\title{
contraction du tube épais circulaire : remarques sur l'influence d'une non linéarité élastique
}

\author{
closure of a thick-walled circular tube : \\ remarks on the influence of non linear elasticity
}

\author{
NGUYEN MINH D. - SCHMITT N. \\ Laboratoire de mécanique des solides * \\ (E.P.-E.N.S.M. Paris, E.N.P.C., \\ Unité associée au C.N.R.S.)
}

\section{Résumé}

On étudie la contraction d'un tube épais, dont le comportement élastique non linéaire est décrit par une loi de Hooke avec module d'Young fonction des invariants des contraintes.

Selon la loi de variation choisie, on met en évidence deux types de répartition de contraintes se traduisant, soit par un durcissement du matériau vers la paroi intérieure, soit par un amollissement accompagné d'un effet de décompression en paroi, comme en plasticité.

Lorsque le module d'Young n'est fonction que de la contrainte moyenne, la répartition des contraintes s'identifie à celle de la solution classique de l'élasticité linéaire.

Ces résultats sont liés au sens de la concavité des courbes effort-déformation obtenues pour des trajets de charge particuliers en compression triaxiale. Ils se reflètent également sur la courbe de convergence du tube, à condition de piloter l'essai par la pression intérieure.

\footnotetext{
Abstract

We study the closure of a non linear elastic thick-walled tube, which behavior is described by a Hooke's law with a Young's Modulus function of the stress invariants.

Depending on the chosen function, two different kinds of stress fields are found out, associated either with a stiffening of the material towards the inner wall of the tube, or with a softening of the material and stress release at the wall, like in plasticity.

When the Young's modulus is but a function of the mean stress, the stress field is identical to the usual linear elastic one.

These results are depending on the concavity sign of stress-strain curves obtained for particular loading pathes in triaxial compression tests. They also can be detected on the closure curves of the tube test, provided that the inner pressure be controlled.
} 


\section{INTRODUCTION}

En mécanique des roches, on a l'habitude, pour simplifier, d'assimiler la phase du comportement précédent la rupture à de l'élasticité linéaire. Mais il est bien connu que le serrage des fissures préexistantes peut conférer aux courbes de réponse du matériau un caractère non linéaire plus ou moins accentué.

Ainsi, dans l'essai de compressibilité (fig. 1.a), ce n'est qu'à partir d'une pression de fermeture des fissures qu'on obtient une réponse linéaire. Cet essai sert ainsi à définir le «volume fissural » (P. HABIB, 1973).

Ce phénomène se retrouve également dans les essais triaxiaux classiques de compression (fig. 1.b). On constate que les courbes «effort-déformation " présentent une concavité tournée «vers le haut », ainsi qu'un raidissement du module de déformation moyen avec laugmentation du confinement. Par contre, le fait que la concavité des courbes soit tournée $x$ vers le bas» au voisinage de la rupture doit plutôt être attribué à des irréversibilités de type « endommagement » ou « plasticitén.

Dans quelle mesure ces phénomènes, souvent négligés, peuvent-ils affecter l'état de contrainte et de défor- mation dans une structure élastique, tel qu'il peut être calculé par la théorie linéaire classique?

Nous allons essayer de discuter de cette question dans le cas particulier d'un tube circulaire épais en contraction, soumis à une pression extérieure uniforme $\mathrm{P}_{\mathrm{e}}$ et une pression intérieure $\mathrm{P}_{1}<\mathrm{P}_{\mathrm{e}}$.

Le problème avait été abordé par J. MANDEL (1962), dans le cadre de l'élasticité non linéaire du second ordre; en exprimant que les courbes effortdéformation présentent une concavité tournée * vers le haut » (fig. 1.b), J. MANDEL montrait que l'intensité de la contrainte tangentielle $\sigma_{\hat{t}}$, en paroi, devait être plus importante que celle qui est obtenue par la solution classique de Kirsch.

Par contre, en exprimant que le « module d'Young » augmente avec le confinement, ou, plus exactement. avec la plus petite contrainte principale en valeur absolue, tout en restant dans le cadre formel de la loi de Hooke, SANTARELLI et al. (1986, 1987) arrivent à un résultat opposé. L'intensité de la contrainte $\sigma_{p}$ est plus faible en paroi (jusqu'à plus de $50 \%$ ), et le maximum peut être atteint à l'intérieur du massif. Ces résultats contradictoires, obtenus par deux modèles de comportement différents, susceptibles de rendre compte d'un même phénomène physique, soulèvent un paradoxe qu'il s'agit de lever.

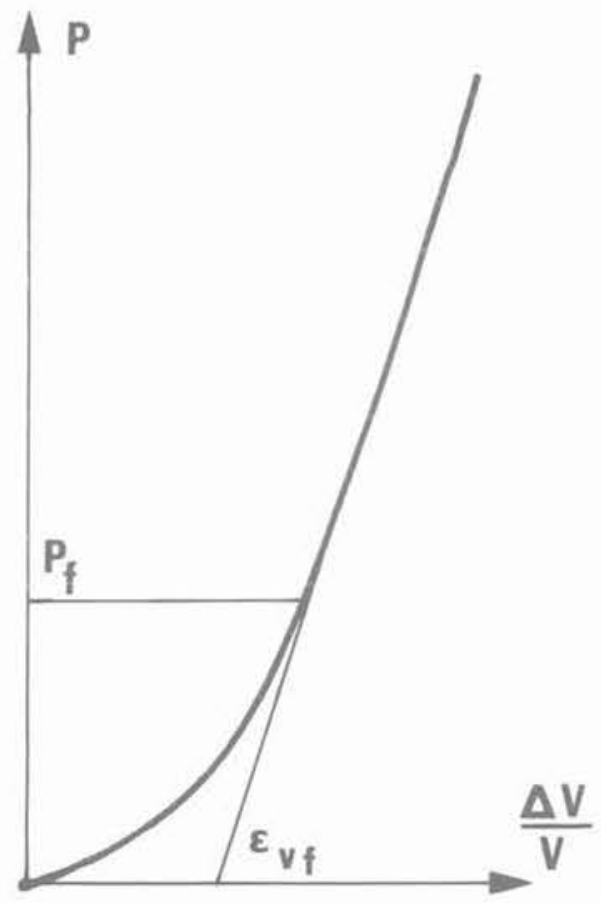

$-\mathbf{a}-$

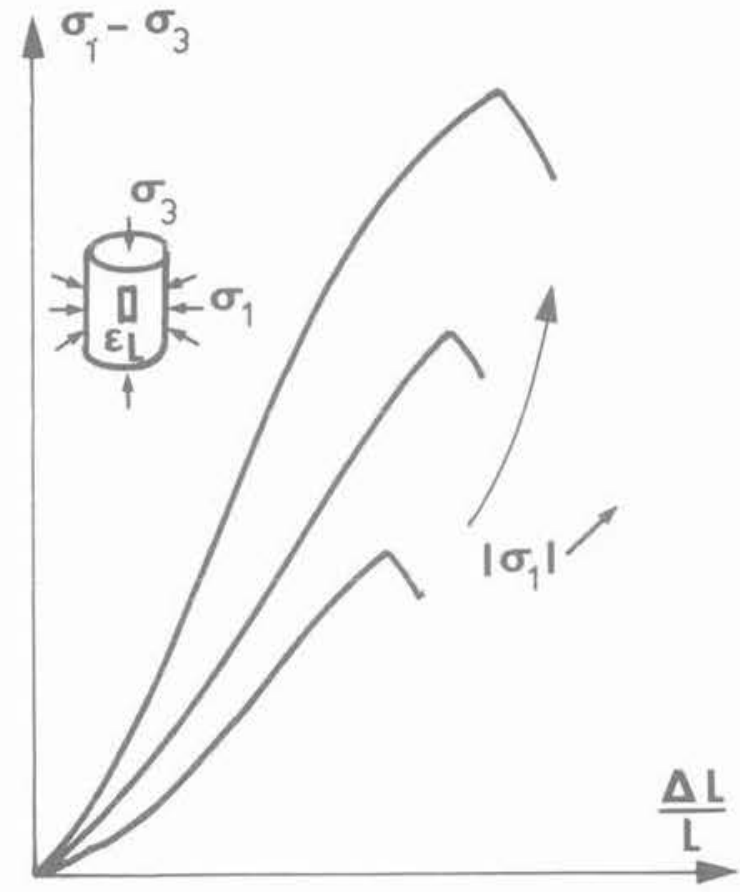

$-\mathbf{b}-$

Fig. 1. - Courbes effort-déformation:

a) Compressibilité,

b) Compression triaxiale.

Fig. 1. - Stress-strain curves:

a) Compressibility test,

b) Triaxial compression test. 


\section{MODÈLE DE COMPORTEMENT}

\subsection{Choix du modèle}

Le modèle adopté par J. MANDEL peut être plus généralement étendu aux termes de déformation d'ordre supérieur. Les calculs sont cependant peu commodes et ne peuvent être qu'approchés. On gardera donc le cadre formel de la loi de Hooke, avec module d'Young variable, adopté par SANTARELLI, et qui avait déjà êté utilisé dans un contexte plus large des déformations finies, où il avait été qualifié de "modèle quasi-linéaire» (FEHRST, 1964).

Comme on considère un matériau isotrope, le "module d'Young E», fonction des contraintes, doit l'être plus précisément des invariants. Les contraintes étant supposées ordonnées :

$$
\sigma_{1}>\sigma_{2}>\sigma_{3} \text { (compression négative), }
$$

on admettra, pour simplifier, que la contrainte intermédiaire n'intervient pas. De ce fait, on doit avoir (J. MANDEL, 1978) :

$$
\mathrm{E}=\mathrm{E}\left(\frac{\sigma_{1}+\sigma_{3}}{2}, \frac{\sigma_{3}-\sigma_{1}}{2}\right)
$$

On ne dispose pas d'essais permettant de préciser la forme (1). Notre analyse étant essentiellement qualita- tive, on se limitera à priori à des formes linéaires pour représenter les tendances observées expérimentale. ment dans les essais triaxiaux de compression, à savoir, un raidissement du matériau au cours du chargement :

$$
E(\sigma)=E_{0} \cdot\left(1+\frac{\alpha}{\sigma_{0}} \cdot \sigma\right)
$$

$\mathrm{E}_{0}$ étant le modèle d'Young a initial »,

$\alpha>0$ : paramètre sans dimension,

$\sigma_{0}<0$ : contrainte caractéristique de compression,

$\sigma<0$ : contrainte équivalente de compression, fonction des invariants.

On se propose de considérer $\sigma$ sous une des formes suivantes :

$$
\begin{array}{ll}
\sigma=\frac{\sigma_{1}+\sigma_{3}}{2} & \text { (contrainte moyenne), } \\
\sigma=\frac{\sigma_{3}-\sigma_{1}}{2} & \text { (contrainte déviatorique), } \\
\sigma=\sigma_{1} & \text { (hypothèse de Santarelli), }
\end{array}
$$$$
\sigma=\frac{\sigma_{1}+\sigma_{3}}{2}+\mathrm{m} \frac{\sigma_{3}-\sigma_{1}}{2} \text { (cas « général »). }
$$

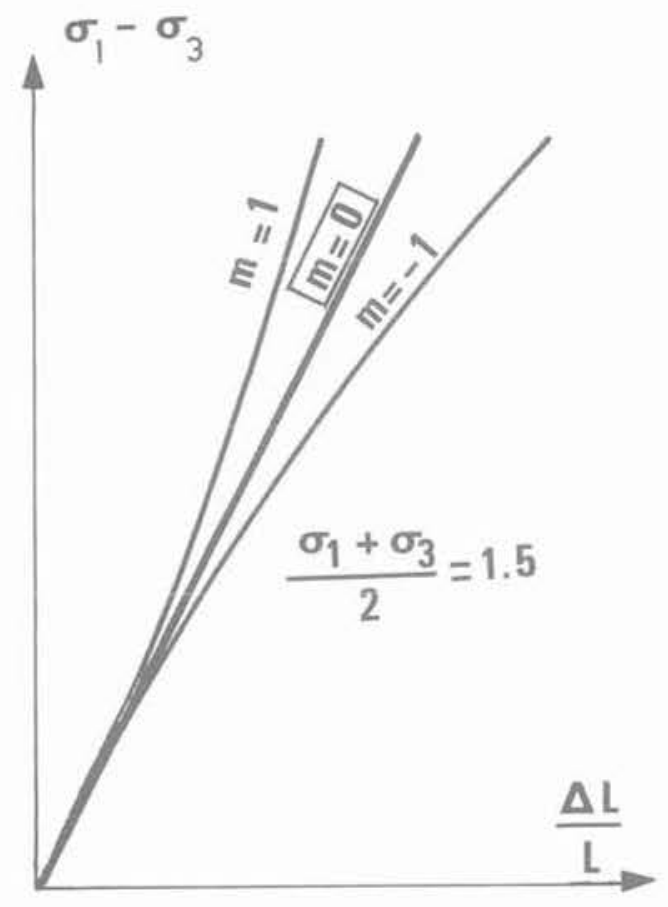

$-\mathbf{a}-$
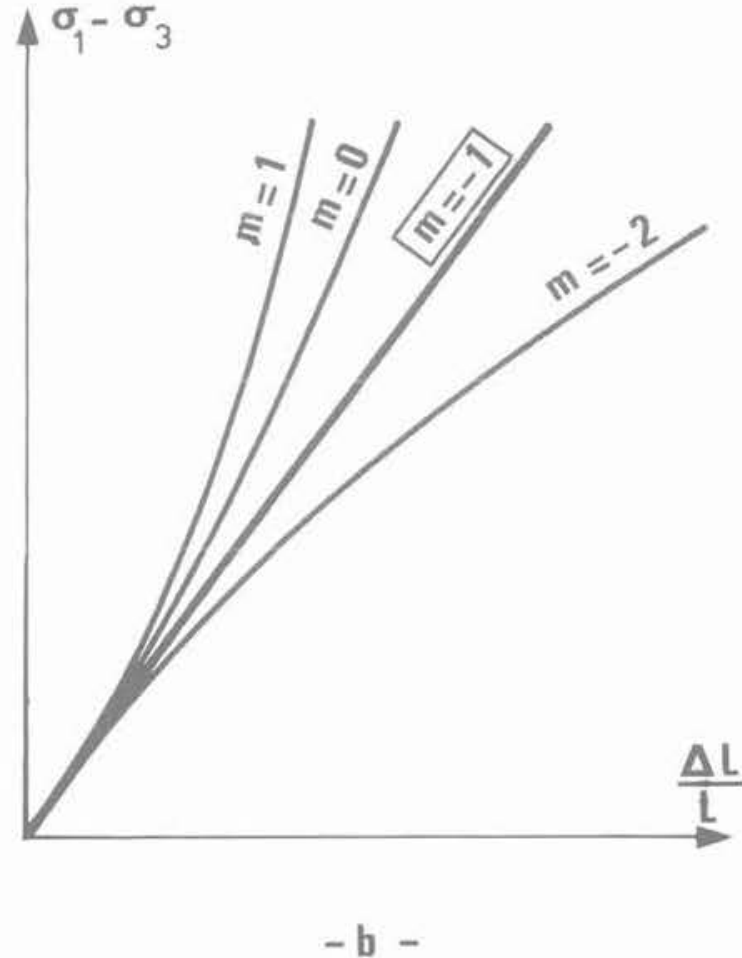

Fig. 2. - Courbes effort-déformation données par le modele :

a) Compression triaxiale avec $\left(\sigma_{1}+\sigma_{3}=C^{\text {te }}\right)$,

b) Compression triaxiale classique $\left(\sigma_{1}=C^{t \theta}\right)$.

Fig. 2. - Stress-strain curves obtained by the model:

a) Triaxial compression with $\left(\sigma_{1}+\sigma_{3}=C^{t \theta}\right)$,

b) Classical triaxial compression $\left(\sigma_{1}=C^{i e}\right)$. 
Le modèle général contient tous les autres modèles; Phypothèse de SANTARELLI apparaît ici comme très particulière $(\mathrm{m}=-1)$.

Il convient de remarquer qu'avec la loi (2), la courbe effort-déformation dans un essai triaxial n'est pas linéaire en général, et sa pente est alors différente de $\mathrm{E}(\sigma)$. Ce paramètre, qu'on continue d'appeler module d'Young dans la mesure où il exprime la proportionnalité entre les tenseurs de contrainte et de déformation, n'a donc plus la signification physique qu'on lui attribue classiquement; il représente en fait un module sécant.

Par ailleurs, il est bien connu que le coefficient de Poisson joue en général un rôle secondaire dans les problèmes élastiques ou élastoplastiques. Sans vouloir sous estimer l'influence d'une variation de ce coefficient avec l'état des contraintes, nous admettrons donc qu'il garde une valeur constante, qu'on choisit égale à $\nu=0,5$. Cette hypothèse d'incompressibilité ne doit pas ôter pour autant à la généralité des phénomènes qualitatifs étudiés.

\subsection{Allure des courbes « effort-déformation»}

Dans un essai triaxial où $\sigma_{1}=\sigma_{2}$, la loi de comportement s'écrit :

$$
\mathrm{E}(\sigma) \cdot \varepsilon_{3}=\sigma_{3}-\sigma_{1}
$$

On distingue deux trajets de charge différents (fig. 2) :

1. Dans l'essai triaxial classique $\left(\sigma_{1}=\mathrm{Cte}\right)$, la courbe effort-déformation est une droite pour $m=-1$ (Hypothèse de SANTARELLI); si m est supérieur à cette valeur, la concavité de la courbe se tourne "vers le haut», et «vers le bas» si $m$ est inférieur.

2. Dans un essai à contrainte moyenne constante $\left(\sigma_{1}+\sigma_{3}\right) / 2=$ Cte, la courbe de réponse est une droite pour $\mathrm{m}=0$; si $\mathrm{m}$ est positif, la concavité se tourne "vers le haut», alors que dans le cas contraire, elle se tourne "vers le bas». Nous utiliserons ici une terminologie particulière en disant dans le premier cas, qu'il y a « durcissement » du matériau et dans le second cas qu'il y a " amollissement".

En l'absence de résultats d'essais spécifiques permettant de préciser le paramètre $m$ du modèle * général *, on considère pour l'instant que le paramètre $\mathrm{m}$ est de signe arbitraire et varie de $-\infty$ à $+\infty$.

Remarque : La relation (2) implique une augmentation de $E$ avec $|\sigma|$. On peut envisager d'écrêter le paramètre $\mathrm{E}$ à la valeur $\mathrm{E}_{0}(1+\alpha)$, par exemple, lorsque $\sigma / \sigma_{0}=1$, ou utiliser entre autre une exponentielle amortie à la place de (2). Cependant, (4) montre que les courbes tendent asymptotiquement vers une droite passant par l'origine; la concavité des courbes doit donc s'inverser à partir d'un certain niveau de chargement, qui se fait d'ailleurs brutalement avec le modèle de E linéaire par morceaux. La simplicité du modèle (4) se heurte à ces inconvénients, qui en montrent les limites. En définitive, on admet que les solutions obtenues avec (2) sont valables dans une certaine plage de variation de $\sigma$.

\section{CALCUL DE LA CONTRACTION DU TUBE CIRCULAIRE ÉPAIS}

La géométrie de ce problème classique à symétrie circulaire est précisée sur la figure 3 . Le tube a un rayon intérieur unité, extérieur $\rho$.

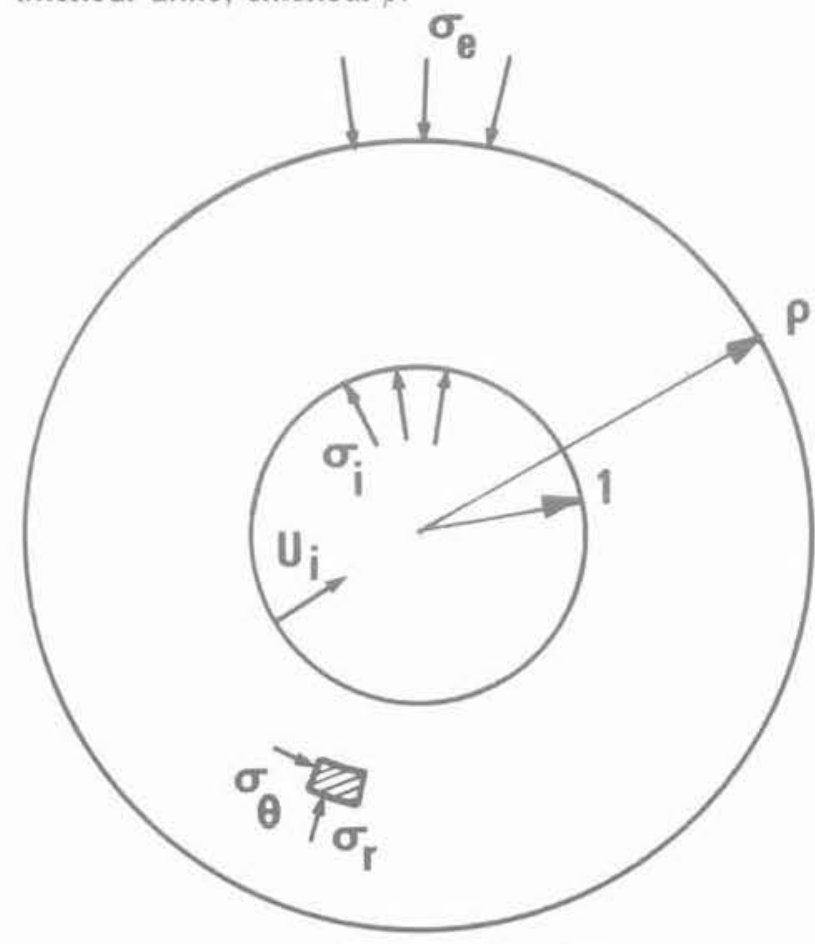

Fig. 3. - Géométrie du problème.

Fig. 3. - Geometry of problem.

Les déformations sont planes, l'état de contraintes initial est isotrope, avec $\sigma_{i}=\sigma_{e}$; la contraction du tube est envisagée soit sous l'action d'une contrainte intérieure $\sigma_{\text {c }}$ croissante (algébriquement), soit sous l'action d'une contrainte extérieure $\sigma_{\text {c }}$ décroissante. On se place dans le cadre des petites transformations.

u étant le déplacement radial d'un point matériel, les équations du problème sont :

$\frac{\mathrm{u}}{\mathrm{r}}+\frac{\partial \mathrm{u}}{\partial \mathrm{r}}=0$

Incompressibilité,

$E^{\prime}(\sigma)=\frac{\mathrm{u}}{\mathrm{r}}=\sigma_{\theta}-\sigma_{\tau}$

Loi de Hooke, où $E^{\prime}(\sigma)=\frac{4}{3} E(\sigma)$

$\frac{\partial \sigma_{r}}{\partial r}=\frac{\sigma_{\theta}-\sigma_{r}}{r}$

Équilibre.

De la première on tire :

$$
\frac{\mathrm{u}}{\mathrm{r}}=\frac{\mathrm{u}_{1}}{\mathrm{r}^{2}}
$$

où $\mathrm{u}_{1}<0$ est le déplacement en paroi, inconnu.

Compte tenu des deux autres équations, on obtient la relation générale :

$$
\frac{\partial \sigma_{r}}{\partial \mathrm{r}}=E^{\prime}(\sigma) \frac{\mathrm{u}_{i}}{\mathrm{r}^{3}}
$$


qui conduit, en fonction du choix de $\mathrm{E}(\sigma)$, aux équations différentielles suivantes, à variables séparées en $\sigma$, et $r$ :

a) $\sigma$ cas général

$\frac{\frac{\alpha}{\sigma_{0}} \mathrm{~d} \sigma_{\mathrm{r}}}{1+\frac{\alpha}{\sigma_{0}} \sigma_{\mathrm{r}}}=\frac{2 \frac{\mathrm{A}}{\mathrm{r}^{3}} \mathrm{dr}}{1-(1+\mathrm{m}) \frac{\mathrm{A}}{\mathrm{r}^{2}}}$

b) $\sigma=\left(\sigma_{\theta}+\sigma_{r}\right) / 2$

$$
\frac{\frac{\alpha}{\sigma_{0}} \mathrm{~d} \sigma_{r}}{1+\frac{\alpha}{\sigma_{0}} \sigma_{r}}=\frac{2 \frac{\mathrm{A}}{\mathrm{r}^{3}} \mathrm{dr}}{1-\frac{\mathrm{A}}{\mathrm{r}^{2}}}
$$

c) $\sigma=\left(\sigma_{\theta}-\sigma_{y}\right) / 2$

$\frac{\alpha}{\sigma_{0}} \mathrm{~d} \sigma_{\mathrm{r}}=\frac{2 \frac{\mathrm{A}}{\mathrm{r}^{3}} \mathrm{dr}}{1-\frac{\mathrm{A}}{\mathrm{r}^{2}}}$

d) $\sigma=\sigma_{r}$

$\frac{\frac{a}{\sigma_{0}} \mathrm{~d} \sigma_{\mathrm{T}}}{a}=2 \frac{\mathrm{A}}{\mathrm{r}^{3}}$

$1+\frac{a}{\sigma_{0}} \sigma_{s}$

avec :

$$
\mathrm{A}=\frac{2 \alpha}{3} \frac{\mathrm{E}_{0}}{\sigma_{0}} \mathrm{u}_{\mathrm{i}}
$$

où $\mathrm{A}$ est une inconnue positive proportionnelle à $\mathrm{u}_{\mathrm{i}}$.

L'intégration de ces équations entre 1 et $\mathrm{r}$ donne $\sigma_{r} ; \sigma_{0}$ se déduit de l'équation d'équilibre; l'inconnue $\mathrm{A}$ se détermine par la condition à la limite $\sigma_{r}(\rho)=\sigma_{e}$, d'où on déduit $u_{1}$ par (7).

Dans le cas « général », (eq. (6.a)), on obtient la solution :

$$
\begin{aligned}
& 1+\alpha-\frac{\sigma_{\tau}}{\sigma_{0}}= \\
& \left(1+\alpha \frac{\sigma_{i}}{\sigma_{0}}\right) \quad\left(1+\frac{(1+\mathrm{m}) \mathrm{A}\left(1-1 / \mathrm{r}^{2}\right)}{1-(1+\mathrm{m}) \mathrm{A}}\right)^{\frac{1}{1+\mathrm{m}}}
\end{aligned}
$$

$$
\begin{aligned}
& 1+\alpha \frac{\sigma_{0}}{\sigma_{0}}= \\
& \left(1+\alpha \frac{\sigma_{t}}{\sigma_{0}}\right) \quad\left(1+\frac{2 \mathrm{~A} / \mathrm{r}^{2}}{1-(1+\mathrm{m}) \mathrm{A} / \mathrm{r}^{2}}\right) \\
& \text { où } \quad \mathrm{A}=\frac{1}{1+\mathrm{m}} \frac{(1+\beta)^{1+\mathrm{m}}-1}{(1+\beta)^{1+\mathrm{m}}-1 / \rho^{2}} \\
& \text { avec } \quad \beta=\frac{\alpha}{\sigma_{0}} \cdot \frac{\sigma_{e}-\sigma_{1}}{\left(1+\alpha \sigma_{1} / \sigma_{0}\right)}
\end{aligned}
$$

\section{DISCUSSION}

La solution générale (8) va permettre de discuter, en fonction de $\mathrm{m}$ (positif ou négatif), de l'évolution de la forme de la solution, tant du point de vue de l'allure des courbes de convergence que de la répartition des contraintes.

\subsection{Courbes de convergence (fig, 4 et 5) :}

Le déplacement en paroi $\mathrm{u}_{i}$, proportionnel à $\mathrm{A}$, dépend du paramètre de chargement $\beta$. L'expression de $\beta$ en fonction des paramètres $\sigma_{e}$ et $\sigma_{1}$ montre que dans un plan $\left\{\left(\sigma_{i}-\sigma_{e}\right),\left|u_{i}\right|\right\}$, la courbe de convergence dépendra du trajet de chargement, c'est-à-dire selon que $\sigma_{e}$ ou $\sigma_{1}$ est la variable commandée.

La discussion portant sur le sens de la concavité de cette courbe est détaillée en annexe 2.

Lorsque le milieu est infini $(\rho=\infty)$, on trouve :

a) Si $\sigma_{1}$ est variable:

- pour $m=0$, la convergence est linéaire ;

- pour $m>0$, la concavité de la courbe est tournée * vers le haut », il y a * raidissement » du tube au cours de la contraction;

- pour $m<0$, on obtient l'effet inverse, le tube "s'amollit $»$.

Dans ce cas, on voit que l'allure des courbes de convergence suivant les valeurs de $m$ reflète celle des courbes effort-déformation du modèle sous compression triaxiale lorsque la contrainte moyenne est maintenue constante.

b) Si $\sigma_{e}$ est variable :

- pour $m=-2$, la convergence est linéaire;

- pour $m>-2$, il y a concavité de la courbe « vers le haut ", c'est-à-dire raidissement du tube, et on obtient bien sûr l'effet inverse pour $\mathrm{m}<-2$.

Néanmoins, les valeurs de $\mathrm{m} \leq-2$ semblent irréalistes, si bien que c'est l'essai avec pression intérieure 


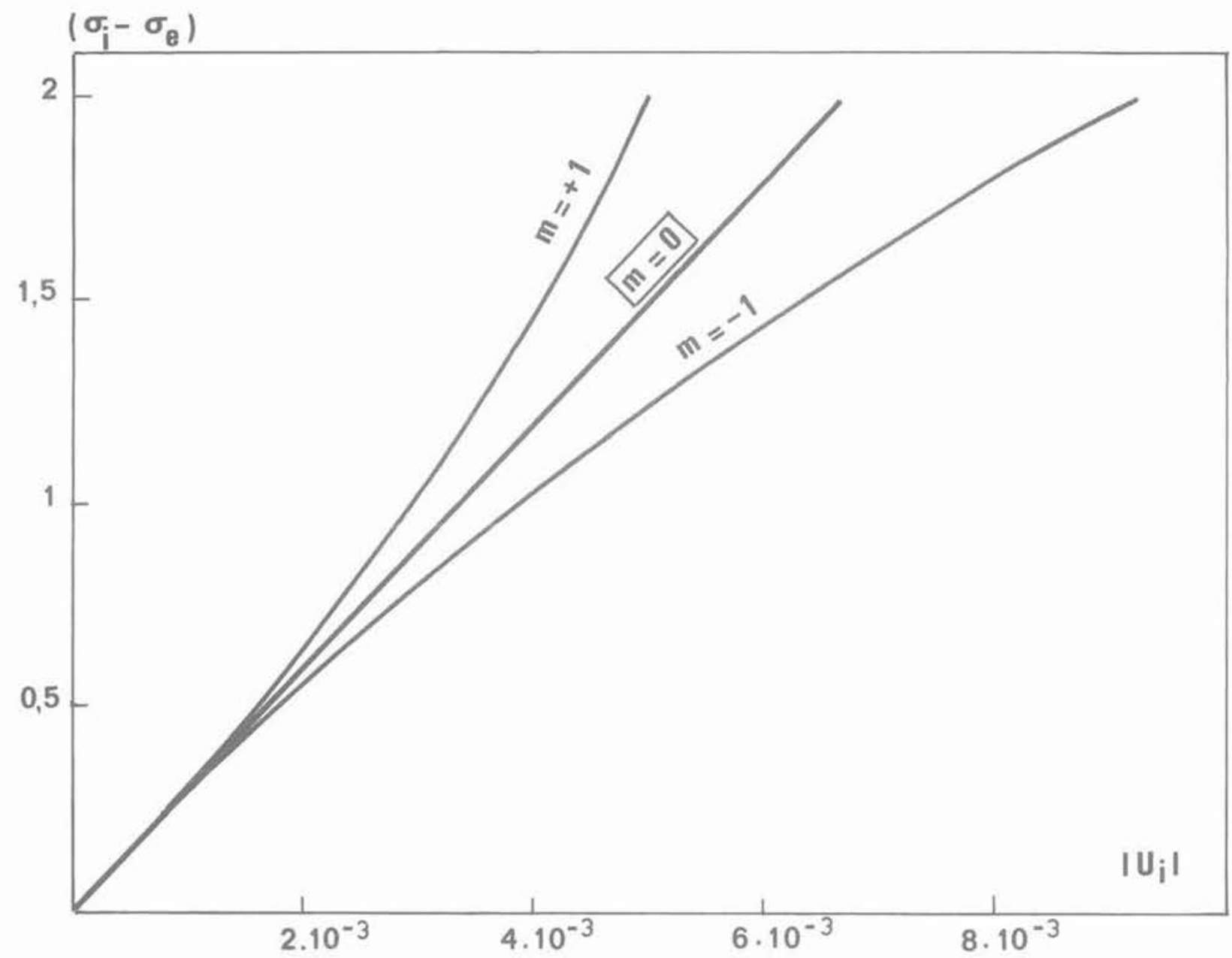

Fig. 4. - Contraction du tube par pilotage de la contrainte intérieure $\sigma_{f}(p=\infty)$.

Fig. 4. - Closure of the tube controlled by internal stress $\sigma_{i}(p=\infty)$.

variable qui peut être intéressant pour préciser le modèle ou indiquer le signe de $\mathrm{m}$.

Évidemment, lorsque p est fini, les résultats précédents restent vrais dans leur ensemble, sous réserve, pour certaines valeurs de $\mathrm{m}$, de conditions sur l'épaisseur du tube ou le niveau de chargement (annexe 2).

D'après l'expression de A (eq. (8)), il est intéressant de remarquer que pour $m<-1$, le paramètre $A$ tend vers l'infini lorsque $\beta$ tend vers une valeur limite $\beta_{0}$, annulant le dénominateur :

$$
\left(1+\beta_{0}\right)^{1+m}=\frac{1}{\rho^{2}}
$$

$\beta_{0}$ apparait ainsi comme une charge limite.

\subsection{Répartition des contraintes (fig. 6)}

La contrainte radiale $\sigma_{\tau}$ est évidemment une fonction décroissante de r, puisque, s'agissant d'une contraction du tube, $\mathrm{u}_{i}<0$, donc par (5), $\mathrm{d} \sigma_{\mathrm{r}} / \mathrm{dr}<0$.
La discussion, détaillée en annexe 3 , porte sur l'existence d'un extremum pour $\sigma_{\theta}$ à l'intérieur du massif. On distingue :

a) Le cas $m=0$ est remarquable car la distribution de contraintes est celle de l'élasticité linéaire (solution de Kirsch). Ce résultat reste valable dans un cadre plus général où le matériau est compressible avec des carac. teristiques élastiques fonctions quelconques de la contrainte moyenne (annexe 4).

b) $\mathrm{m}>0$. La contrainte $\sigma_{\theta}$ conserve la même allure que précédemment : l'intensité maximale est atteinte en paroi, où elle est supérieure à celle de la solution classique ; parallèlement, il y a durcissement du matériau vers la paroi intérieure, car $E^{\prime}(\sigma)$ est fonction décroissante de r (annexe 2).

c) $\mathrm{m}<0$. Au cours du chargement, la contrainte $\sigma_{\hat{\theta}}$ a d'abord la même allure que dans les cas précédents ; si le chargement se poursuit, il apparaît un extremum de $\sigma_{\theta}$ se déplaçant vers l'intérieur du massif au point $\mathrm{r}_{1}^{2}=(1-\mathrm{m}) \mathrm{A}$; si le tube a une épaisseur finie, l'extremum peut même * sortir » du tube $\left(r_{1}^{2}>\rho^{2}\right)$, de sorte que $\sigma_{\theta}$ devienne une fonction strictement décroissante de $r$. 


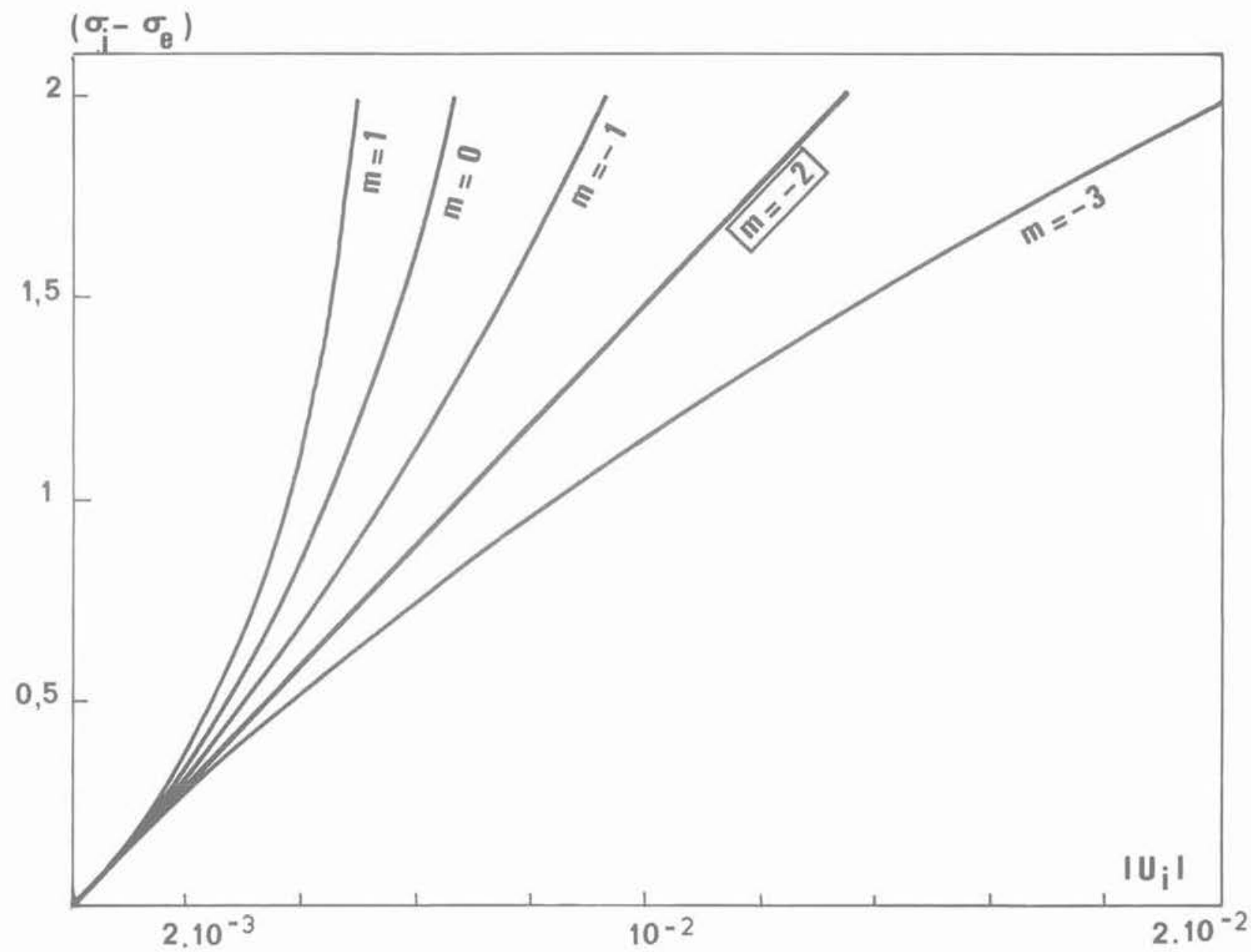

Fig. 5. - Contraction du tube par pilotage de la contrainte extérieure $\sigma_{\mathrm{e}}(p=\infty)$.

Fig. 5. - Closure of the tube controlled by external stress $\sigma_{\mathrm{e}}(p=\infty)$.

La contrainte en paroi est ici inférieure en intensité à celle de la solution classique; parallèlement, il y a amollissement du matériau vers la paroi intérieure, $E^{\prime}(\sigma)$ étant ici fonction croissante de $\mathrm{r}$.

Ces différents aspects indiquent certaines analogies de comportement avec l'élastoplasticité.

Des résultats similaires avaient d'ailleurs été mis en évidence dans un contexte tout à fait différent par SCHMITT (1987). Celui-ci avait étudié l'équilibre d'une galerie circulaire chauffée dans un massif élastique dont le module d'Young était fonction décroissante de la température, donc fonction croissante du rayon.

Il est intéressant de noter dans le cas où $\rho=\infty$ que l'intensité du pic de $\sigma_{\theta}$ reste constante si $\sigma_{1}$ est la variable commandée alors qu'elle augmente avec le chargement dans le cas contraire; en effet :

$\left(1+\alpha \cdot \sigma^{M} / \sigma_{0}\right)=\frac{1+\alpha \cdot \sigma_{e} / \sigma_{0}}{1+\alpha \cdot \sigma_{e}^{*} / \sigma_{0}}$

$$
\cdot\left(1+\alpha \cdot \sigma_{i}^{*} / \sigma_{0}\right) \cdot(1-1 / m)
$$

où $\sigma_{\theta}^{M}$ est la valeur de l'extremum, et $\left(^{*}\right)$ se rapporte au paramètre de charge relatif à l'apparition de l'extremum en paroi.

Comme par ailleurs, la valeur absolue de la contrainte moyenne croît avec le rayon (annexe $3, \S 2$ ), ceci implique qu'avec un critère de rupture ou de plasticité de type "courbe intrinsèque ", la limite élastique est toujours atteinte en paroi lorsque $\left|\sigma_{i}\right|$ décroît, alors que si $\left|\sigma_{e}\right|$ croit, elle peut être atteinte soit en paroi, soit, éventuellement, à l'intérieur du massif dans la zone $1<r \leq r_{1}$.

\section{CONCLUSION}

En utilisant un modèle de comportement assez simple, basé sur la relation de HOOKE avec un "module d'Young "variable, fonction de l'état de contraintes, nous avons discuté de l'influence de la non-linéarité élastique sur l'état d'équilibre dans un tube circulaire épais en contraction. 


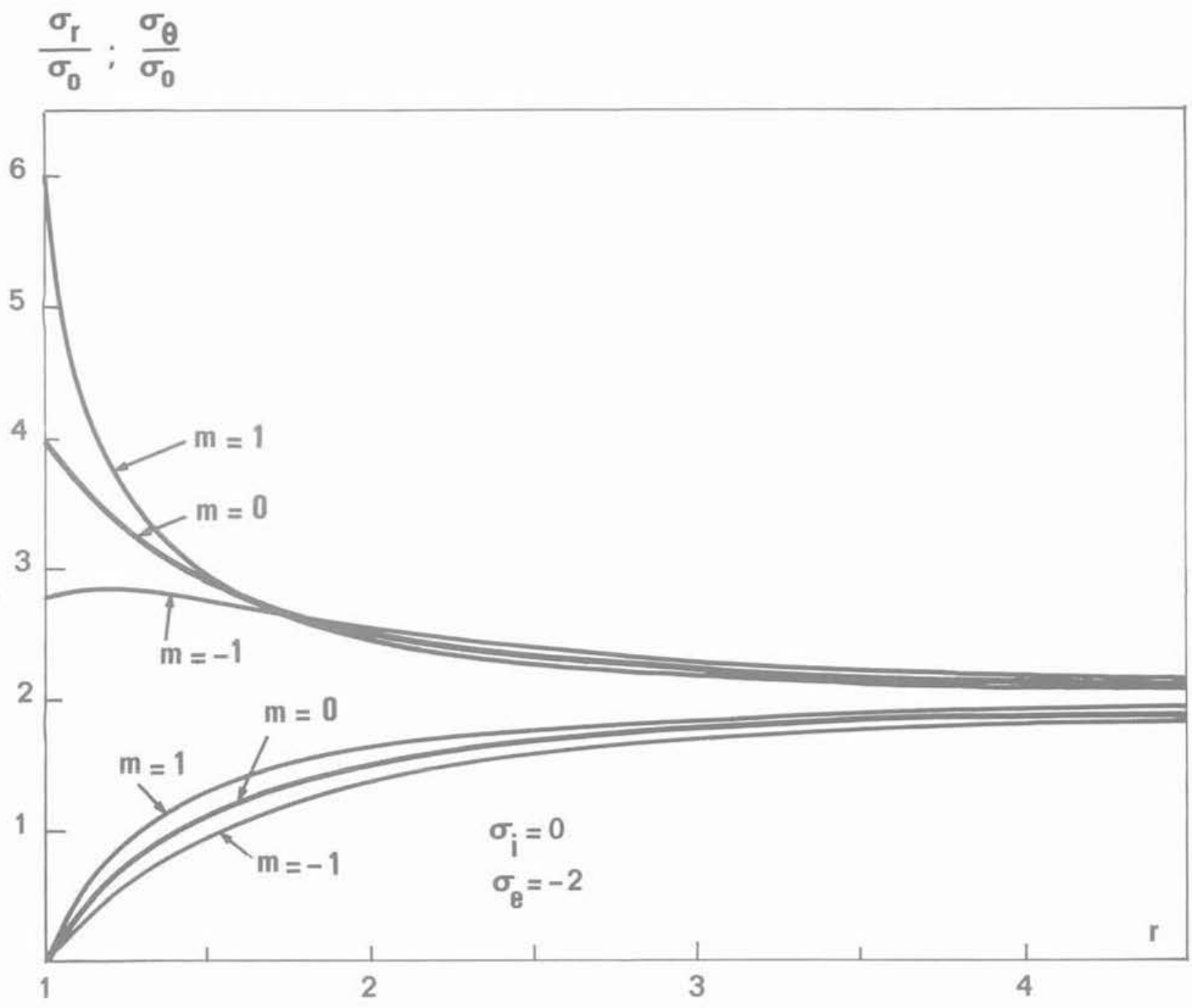

Fig. 6. - Répartition des contraintes pour le cylindre infini.

Fig. 6. - Stress distribution for infinite hollow cylinder.

A condition de se référer à des essais triaxiaux de compression à contrainte moyenne imposée $\left(\sigma_{1}+\sigma_{3}=\right.$ Cte), on met en évidence la corrélation étroite entre lallure des courbes effort-déformation correspondant à ces essais et la distribution des contraintes dans le tube : - Si la concavité des courbes est tournée vers le haut ( $\mathrm{m}>0$ ), c'est-à-dire si la raideur du matériau augmente en cours de compression, il résulte, pour le tube, un durcissement du matériau vers la paroi interne, où la contrainte tangentielle atteint son intensité maximale, supérieure à celle de la solution de $\mathrm{KIRSCH}$. Ce résultat rejoint celui de J. MANDEL.

- A l'inverse, si la raideur du matériau diminue au cours de la compression $(m<0)$, comme dans le cas particulier de lhypothèse de SANTARELLI $(m=-1)$, il résulte pour le tube un amollissement du matériau vers la paroi interne, où la contrainte tangentielle devient inférieure en intensité à celle de la solution classique. Parallèlement, l'extremum de la contrainte tangentielle est rejeté à l'intérieur du massif, traduisant un effet de décompression en paroi. La similitude avec l'élastoplasticité s'étend à la notion de charge limite, lorsque la concavité « vers le bas " des courbes effort-déformation des essais triaxiaux à contrainte moyenne constante est très marquée.

- Enfin, lorsque les courbes efforts-déformation sont linéaires $(m=0)$, la distribution des contraintes dans le tube s'identifie avec celle de la solution classique de l'élasticité linaire. Le module d'Young, fonction de la contrainte moyenne, est alors constant dans le tube. La non-linéarité élastique se manifeste ici par une réponse différente au niveau de la convergence du tube suivant le trajet de charge adopté : ainsi, si la pression intérieure est la variable commandée, la convergence évolue de façon presque linéaire; par contre, si c'est la pression extérieure qui est la variable commandée, la convergence est non linéaire, par suite de l'augmentation du module d'Young.

Il apparaît ainsi, que pour des roches avec une élasticité non linéaire marquée, il est nécessaire de prévoir un ensemble de procédures expérimentales adaptées, pour identifier la loi de variation du module d'Young.

Pour les sols, les essais triaxiaux à pression moyenne constante montrent des déformations pratiquement 
linéaires au début du chargement (ou du déchargement) (LUONG, 1980), c'est-à-dire dans le cas présent que la solution de $\mathrm{KIRSCH}$ resterait valable pour un trou circulaire dans un sol.

$\mathrm{Ne}$ disposant pas encore suffisamment de résultats expérimentaux pour les roches, il est difficile d'émettre à ce sujet autre chose que des conjectures. Il semble raisonnable d'attribuer l'essentiel du phénomène de serrage des roches à l'effet de la contrainte moyenne, ce qui revient à se limiter à des faibles valeurs de $\mathrm{m}$, quel que soit son signe. Mais, il ne faut pas exclure la possibilité d'une plus forte influence du déviateur des contraintes pour certains types de matériaux.

\section{RÉFÉRENCES}

FEHRST S. (1964), Examination of quasi-linear elasticity, pp. 252-260. Second order effects in Elasticity, Plasticity and Fluid Dynamics, Int. Symp. Haifa, Israël, 1962. Pergamon Press Book, Mc Dilan Comp., N.Y. HABIB P. (1973), Précis de Géotechnique, Dunod Ed.

LUONG M.P. (1980), Stress-strain aspects of cohesionless soils under cyclic and transient loading. Proc. Int. Symp. on Soils under Cyclic and Transient Loading, Swansea, 7-11 Jan

MANDEL J. (1959), Les calculs en matière de pression des terrains, revue de l'Industrie minérale, pp. 78-92.

MANDEL J. (1978), Propriétés mécaniques des matériaux, Eyrolles Éd., Paris.

SANTARELLI F.J., BROWN E.T., MAURY V. (1986), Analysis of borehole stresses using pressure dependent, linear elasticity. Technical Note, Int. J. Rock Mech. Min. Sci. \& Geom. Abstr., vol. 23, n 6 , pp. 445-449.

SANTARELLI F.J., BROWN E.T. (1987), Performance of deep wellbores in rock with a confining pressure-dependent elastic modulus, 6th Cong. Int. SMIR, Montréal, 1987, Proceedings.

SCHMITT N. (1987), Étude du comportement thermomécanique du charbon dans le cadre de la gazéification souterraine. Analyse de la stabilité de la liaison de gazéification, Thèse de doctorat de l'Université Paris VI, 10 juillet 1987

Annexe 1 : solutions obtenues selon le choix particulier du paramètre $\sigma$

La solution du * cas général » où $\sigma$ est fonction du paramètre $m$ est donnée en (8). Pour les autres cas envisagés en (7), nous donnons, ci-après, les solutions qui s'obtiennent aussi par (8) pour des valeurs particulières de $\mathrm{m}$, éventuellement par passage à la limite.

1. Cas $m=0$ ou $\sigma=\left(\sigma_{\theta}+\sigma_{\mathrm{r}}\right) / 2$ (contrainte moyenne) :

La distribution des contraintes est identique à celle de l'élasticité linéaire (solution de KIRSCH). Ce résultat reste valable quelle que soit la forme $E(\sigma)$ (annexe 4).

$$
\begin{aligned}
& \sigma_{\mathrm{r}}=\sigma_{\mathrm{l}}+\left(\sigma_{e}-\sigma_{\mathrm{i}}\right) \frac{1-1 / \mathrm{r}^{2}}{1-1 / \rho^{2}} \\
& \sigma_{\theta}=\sigma_{\mathrm{i}}+\left(\sigma_{e}-\sigma_{1}\right) \frac{1+1 / \mathrm{r}^{2}}{1-1 / \rho^{2}} \\
& \mathrm{~A}=\frac{\alpha}{\sigma_{0}} \cdot \frac{\left(\sigma_{e}-\sigma_{i}\right)}{1+\alpha \frac{\sigma_{e}}{\sigma_{0}}-\left(1+\alpha \frac{\sigma_{i}}{\sigma_{0}}\right) / \rho^{2}}
\end{aligned}
$$

On remarquera que dans ce cas, la contrainte moyenne est constante à l'intérieur du tube :

$$
\sigma=\frac{\left(\sigma_{\theta}+\sigma_{r}\right)}{2}=\frac{\sigma_{e}-\sigma_{i} / \rho^{2}}{1-1 / \rho^{2}}
$$

2. Cas $\sigma=\left(\sigma_{\theta}-\sigma_{x}\right) / 2$ (contrainte déviatorique):

$$
\left.\begin{array}{l}
\sigma_{\tau}=\sigma_{1}+\frac{\sigma_{0}}{\alpha} \log \left(\frac{1-\mathrm{A} / \mathrm{r}^{2}}{1-\mathrm{A}}\right) \\
\sigma_{\theta}=\sigma_{1}+\frac{\sigma_{0}}{\alpha}\left\{\log \left(\frac{1-\mathrm{A} / \mathrm{r}^{2}}{1-\mathrm{A}}\right)+\frac{2 \mathrm{~A} / \mathrm{r}^{2}}{1-\mathrm{A} / \mathrm{r}^{2}}\right\} \\
\mathrm{A}=\frac{\exp \left(\frac{\alpha\left(\sigma_{e}-\sigma_{i}\right)}{\sigma_{0}}\right)-1}{\exp \left(\frac{\alpha\left(\sigma_{e}-\sigma_{t}\right)}{\sigma_{0}}\right)-\frac{1}{\rho^{2}}}
\end{array}\right]
$$

Cette solution s'obtient aussi par la solution générale, par passage à la limite $\mathrm{m} \rightarrow+\infty$, à condition de remplacer le paramètre $\sigma_{0}$ par $\mathrm{m} \sigma_{0}$. Ainsi, dans l'expression de A de la solution générale (8), le terme $(1+\beta)^{1+m}$ tend vers $\exp \left[\alpha\left(\sigma_{e}-\sigma_{1}\right) / \sigma_{0}\right]$. Remarquons que le cas $\mathrm{m} \rightarrow-\infty$ correspondrait à $\sigma=\left(\sigma_{r}-\sigma_{\theta}\right) / 2$

3. Cas $\sigma=\sigma_{\uparrow}$ ou $m \rightarrow-1$ :

$$
\begin{aligned}
& 1+\alpha \frac{\sigma_{r}}{\sigma_{0}}= \\
& \left(1+\alpha \frac{\sigma_{1}}{\sigma_{0}}\right) \exp \left[\mathrm{A}\left(1-\frac{1}{\mathrm{r}^{2}}\right)\right] \\
& 1+\alpha \frac{\sigma_{0}}{\sigma_{0}}= \\
& \left(1+\alpha \frac{\sigma_{1}}{\sigma_{0}}\right)\left(1+\frac{2 \mathrm{~A}}{\mathrm{r}^{2}}\right) \exp \left[\mathrm{A}\left(1-\frac{1}{\mathrm{r}^{2}}\right)\right] \\
& \mathrm{A}=\frac{1}{1-\frac{1}{\rho^{2}}} \log (1+\beta)
\end{aligned}
$$

Cette solution peut s'obtenir à partir de la solution générale par passage à la limite $m \rightarrow-1$. Ainsi, 
dans l'expression de $A$ de la solution (8), le terme $(1+\beta)^{1+m}$ est équivalent à $(1+m) \log (1+\beta)$; dans l'expression de $\sigma$ dans (8), le terme affecté de l'exposant $1 /(1+\mathrm{m}$ ) (infiniment grand) équivaut à : $\exp \left(A\left(1-1 / r^{2}\right)\right)$.

\section{Annexe 2: concavité des courbes de conver- gence}

On discute, en fonction du paramètre $\mathrm{m}$ de la solution générale (8), de la concavité de la courbe de conver. gence dans le plan $\left(\sigma_{1}-\sigma_{\mathrm{e}}\right)$, $\left|\mathrm{u}_{1}\right|$. Il est équivalent de considérer, à la place de $\left|\mathrm{u}_{i}\right|$, le paramètre A positif, qui lui est proportionnel.

On distingue le cas où $\sigma$ est variable $(\sigma$, croît à partir de $\left.\sigma_{e}\right)$, du cas où $\sigma_{e}$ est variable $\left(\sigma_{e}\right.$ décroît à partir de $\left.\sigma_{1}\right)$.

\section{Remarque préliminaire :}

Dans l'expression de A (eq (8)), le dénominateur doit rester positif, d'où :

$$
(1+\beta)^{1+m}>\frac{1}{\rho^{2}}
$$

Cette inégalité est triviale si $\mathrm{m}+1>0$, mais dans le cas contraire, elle donne une borne supérieure $\beta_{0}$ au paramètre de charge $\beta$, pour laquelle $A$ tend vers l'infini : cette valeur $\beta_{0}$ équivaut alors à une charge limite pour la structure.

\section{2. $\sigma_{1}$ est la variable commandée :}

$\sigma_{e}$ étant constante, la concavité de la courbe de convergence dépend du signe de :

$$
\begin{aligned}
& \frac{1}{\mathrm{C}} \frac{\mathrm{d}^{2} \mathrm{~A}}{\mathrm{~d} \sigma_{t}^{2}}=-\mathrm{m}\left\{(1+\beta)^{1+m}-\frac{\omega}{\rho^{2}}\right\} \\
& \text { où } \quad \omega=-\frac{\mathrm{m}+2}{\mathrm{~m}}
\end{aligned}
$$

et $\mathrm{C}$ est une quantité strictement positive :

$$
C=\frac{\frac{\alpha^{2}}{\sigma_{0}^{2}}\left(1-\frac{1}{\rho^{2}}\right)(1+\beta)^{1+m}\left(1+\frac{\alpha \sigma_{1}}{\sigma_{0}}\right)^{-2}}{\left((1+\beta)^{1+m}-\frac{1}{\rho^{2}}\right)^{3}}
$$

d'où l'on déduit :

a) $\rho=\infty$ le signe de $\mathrm{d}^{2} \mathrm{~A} / \mathrm{d} \sigma_{\mathrm{j}}^{2}$ est l'inverse de celui de $\mathrm{m}$ :

- si $\mathrm{m}=0$, la convergence est linéaire ;

- $m>0$, la concavité est tournée « vers le haut », le tube se "rigidifie » au cours de sa convergence;

- $\mathrm{m}<0$, la concavité se tourne "vers le bas ", le tube " s'amollit " au cours de sa convergence. b) $\rho$ fini : on vérifie que les propriétés précédentes restent conservées dans leur ensemble, sauf pour $-1<\mathrm{m}<0$, où elles le sont sous certaines conditions.

Dans ce cas, en effet, on a $\omega>1$; d'après (A.5), la concavité n'est tournée * vers le bas », comme pour $\rho=\infty$, que si $\rho^{2}>\omega$, c'est-à-dire, si l'épaisseur du tube est suffisante; s'il n'en est pas ainsi $\left(\rho^{2}<\omega\right)$, la concavité initiale de la courbe de convergence est tournée "vers le haut », mais peut changer de sens si le chargement est suffisant ; c'est-à-dire si :

$$
(1+\beta)^{1+m}>\frac{\omega}{\rho^{2}}
$$

Par ailleurs, pour $m=0$, la concavité se tourne vers le bas, mais reste faible, car le rayon de courbure est proportionnel à $\rho^{2}$ (eq. (A.5)).

\section{3. $\sigma_{e}$ est la variable commandée :}

$\sigma_{i}$ étant constante, la concavité de la courbe de convergence dépend du signe de :

$$
\begin{aligned}
& \frac{1}{C^{\prime}} \frac{d^{2} A}{d \sigma_{e}^{2}}=-m\left\{\frac{1}{\rho^{2}}-\omega(1+\beta)^{1-m}\right\} \\
& \text { où } \quad \omega=-\frac{m+2}{m}
\end{aligned}
$$

et $C^{\prime}$ une quantité strictement positive :

$$
C^{\prime}=C /(1+\beta)^{2} \quad \text { (eq. (A.5)). }
$$

D'où l'on déduit, de la même façon que précédemment :

a) $p=\infty$ :

- si $m=-2$, la courbe de convergence est linéaire;

- si $\mathrm{m}>-2$, sa concavité est tournée « vers le haut : :

- si $\mathrm{m}<-2$, sa concavité est tournée « vers le bas .

b) Si $p$ est fini : les propriétés précédentes restent vraies, sauf lorsque $-2<\mathrm{m}<-1$, pour laquelle la concavité de la courbe ne reste tournée * vers le haut * que si l'épaisseur du tube est suffisante:

$$
\left.\rho^{2}>\frac{1}{\omega} \text { où } \omega \in\right] 0,1[
$$

et à condition que le chargement ne soit pas trop important :

$$
(1+\beta)^{1+m}<\frac{1}{\rho^{2} \omega} .
$$

Si le chargement dépasse la valeur limite correspondante, ce qui est possible puisque (A.9) n'est pas en contradiction avec (A.4), la concavité change de sens et se tourne "vers le bas $»$.

Enfin, si (A.8) n'est pas vérifiée, c'est-à-dire si l'épaisseur du tube est insuffisante, la concavité est toujours tournée « vers le bas ». 


\section{Annexe 3 : état des contraintes dans le massif}

On se reporte, comme précédemment, à la solution générale. Rappelons que $\beta$ (eq. (8)) joue le rôle d'un paramètre de chargement.

1. Existence d'un extremum pour la contrainte tangentielle $\sigma_{\theta}$ :

D'après (8), la dérivée de la contrainte s'écrit :

$$
\begin{aligned}
\frac{1}{\mathrm{D}} \frac{\mathrm{d} \sigma_{\theta}}{\mathrm{dr}}=\frac{\sigma_{0}}{\alpha} \quad\left(-1+(1-\mathrm{m}) \frac{\mathrm{A}}{\mathrm{r}^{2}}\right) \\
\text { où } \mathrm{D}=\frac{2 \mathrm{~A} / \mathrm{r}^{3}\left(1+\alpha \sigma_{\mathrm{r}} / \sigma_{0}\right)}{\left(1-(1+\mathrm{m}) \mathrm{A} / \mathrm{r}^{2}\right)^{2}}
\end{aligned}
$$

D étant une quantité positive au départ du chargement (A proche de zéro), $\mathrm{d} \sigma_{\theta} / \mathrm{dr}>0$ : c'est l'allure classique de la contrainte tangentielle $\sigma_{\theta}$ dans la solution élastique linéaire; le maximum d'intensité est alors atteint en paroi.

L'extremum de $\sigma_{\theta}$ peut avoir lieu à l'intérieur du massif si la valeur de $\mathrm{r}^{2}$ annulant (A.10) est supérieure à 1 :

$$
r_{1}^{2}=(1-m) A>1
$$

$r_{1}^{2}$ peut encore s'écrire :

$$
\begin{aligned}
r_{1}^{2}=\lambda(m) g_{m}(\beta) \text { où } \lambda(m) & =\frac{1-m}{1+m} \\
\text { et } g_{m}(\beta) & =\frac{(1+\beta)^{1+m}-1}{(1+\beta)^{1+m}-\frac{1}{\rho^{2}}}
\end{aligned}
$$

alors :

a) Si $m \geq 0$ :

$$
\mathrm{r}_{1}^{2}<0 \text { car } \lambda(\mathrm{m}) \leq 1 \text { et } \mathrm{g}_{\mathrm{m}}(\beta)<1
$$

Il ne peut y avoir d'extremum pour $\sigma_{\theta}$ à l'intérieur du massif.

b) Si $m<0$ :

$\diamond \mathrm{m} \in]-1,0[$; alors $\lambda(\mathrm{m}) \in[1,+\infty]$

$\mathrm{g}_{\mathrm{m}}(\beta)$, initialement nulle, croît avec $\beta$ et tend vers 1 pour $\beta \rightarrow \infty$. Il existe donc un chargement $\beta_{1}$, fini, pour lequel $r_{1}^{2}=1$, et à partir duquel $r_{1}^{2}$ augmente jusqu'à la valeur limite $\lambda(\mathrm{m})$.

$\diamond m \in]-\infty,-1 ;$ alors $\lambda(m) \in]-\infty,-1$ [

$\mathrm{g}_{\mathrm{m}}(\beta)$, initialement nulle, est une fonction décroissante de $\beta$, et tend vers $-\infty$ lorsque $\beta$ tend vers la "charge limite " $\beta_{0}:\left(1+\beta_{0}\right)^{1+m}=1 / \rho^{2}$; on peut donc toujours trouver une valeur du chargement $\beta_{1}<\beta_{0}$, pour laquelle $\mathrm{r}_{1}^{2}$ augmente indéfiniment.

En résumé, quel que soit $m<0$, on peut trouver une charge $\beta_{1}$ au-delà de laquelle $\sigma_{\theta}$ admet un extremum se déplaçant vers l'intérieur du tube lorsque le chargement se poursuit. Lorsque le rayon extérieur $\rho$ est fini, il peut arriver, pour certaines valeurs de $\mathrm{m}$, et pour des chargements suffisants, que $r_{1}>\rho$ : la contrainte $\sigma_{\phi}$ devient alors une fonction strictement croissante de $r$.
2. Sens de variation $d u$ * module d'Young $* E(\sigma)$ avec $r$ :

On obtient, par (6) et (8) :

$$
\left.\begin{array}{l}
\frac{\alpha}{\sigma_{0}} \frac{\mathrm{d}}{\mathrm{dr}}\left(\frac{\sigma_{\mathrm{r}}+\sigma_{f}}{2}\right)=\frac{-\mathrm{m} \frac{\mathrm{A}}{\mathrm{r}^{2}} \mathrm{D}}{1-(1+\mathrm{m}) \frac{\mathrm{A}}{\mathrm{r}^{2}}} \\
\frac{\alpha}{\sigma_{0}} \frac{\mathrm{d}}{\mathrm{dr}}\left(\frac{\sigma_{\theta}-\sigma_{\mathrm{r}}}{2}\right)=\frac{\left(-1+\frac{\mathrm{A}}{\mathrm{r}^{2}}\right) \mathrm{D}}{1-(1+\mathrm{m}) \frac{\mathrm{A}}{\mathrm{r}^{2}}}
\end{array}\right]
$$

où $\mathrm{D}$ est strictement positif (eq. (A.10)) ; d'où :

$$
\frac{d E^{\prime}(\sigma)}{d r}=\frac{-m D}{1-(1+m) \frac{A}{r^{2}}}
$$

Le dénominateur étant strictement positif, le signe de cette dérivée est l'inverse de celui de $\mathrm{m}$.

Lorsque $m$ est positif, $E$ est donc maximal en parol et décroît vers l'intérieur du massif. On a le contraire lorsque $\mathrm{m}<0$.

Remarquons, d'après (A.12), qu'il en est de même pour la valeur absolue de la contrainte moyenne $\left(\sigma_{\tau}+\sigma_{\theta}\right) / 2$.

\section{Contrainte tangentielle en paroi :}

D'après (8) :

$$
\sigma_{\beta}(1)=\sigma_{1}+\frac{2\left(1+\alpha \frac{\sigma_{i}}{\sigma_{0}}\right)\left[(1+\beta)^{1+m}-1\right]}{\left(1-\frac{1}{\sigma_{2}}\right) \alpha(1+m)}
$$

On vérifie que cette quantité est fonction décroissante de $\mathrm{m}$.

Comme la solution en contraintes s'identifie à celle de KIRSCH pour $\mathrm{m}=0$, l'intensité de $\sigma_{\theta}(1)$ est donc supérieure à la valeur classique si $\mathrm{m}$ est positif, et lui est inférieure si $\mathrm{m}$ est négatif.

Annexe 4: solution dans le cas ou $\sigma$ est la contrainte moyenne et $\mathrm{E}(\sigma)$ une fonction quelconque. Cas du matériau compressible

Nous allons montrer que la solution en contraintes, identique à celle de KIRSCH dans le cas où $E(\sigma)$ est une fonction linéaire, l'est aussi dans le cas où $E(\sigma)$ est quelconque. Pour cela, il suffit de montrer que $\sigma$ est constante. 
Compte tenu de l'équation d'équilibre :

$$
\sigma=\sigma_{\mathrm{r}}+\frac{1}{2} \mathrm{r} \frac{\partial \sigma_{\mathrm{r}}}{\partial \mathrm{r}}
$$

soit :

$$
\frac{\mathrm{d} \sigma}{\mathrm{dr}}=\frac{3}{2} \frac{\partial \sigma_{r}}{\partial \mathrm{r}}+\frac{1}{2} \mathrm{r} \frac{\partial^{2} \sigma_{t}}{\partial \mathrm{r}^{2}}
$$

or :

$$
\frac{\partial \sigma_{t}}{\partial \mathrm{r}}=\mathrm{E}^{\prime}(\sigma) \frac{\mathrm{u}_{i}}{\mathrm{r}^{3}} \text { (eq. }
$$

et :

$$
\mathrm{r} \frac{\partial^{2} \sigma_{\mathrm{r}}}{\partial \mathrm{r}^{2}}=-3 \mathrm{E}^{\prime}(\sigma) \frac{\mathrm{u}_{1}}{\mathrm{r}^{3}}+\frac{\mathrm{dE}}{\mathrm{d} \sigma} \frac{\mathrm{d} \sigma}{\mathrm{dr}} \frac{\mathrm{u}_{\mathrm{i}}}{\mathrm{r}^{2}}
$$

Les trois dernières équations donnent alors

$$
\frac{\mathrm{d} \sigma}{\mathrm{dr}} \cdot\left(2-\frac{\mathrm{u}_{\mathrm{i}}}{\mathrm{r}^{2}} \frac{\mathrm{d} \mathrm{E}^{\prime}(\sigma)}{\mathrm{d} \sigma}\right)=0
$$

Or, $\mathrm{d} \mathrm{E}^{\prime}(\sigma) / \mathrm{d} \sigma$ est une fonction donnée de $\sigma$, essentiellement bornée.

Par ailleurs, $\mathrm{u}_{\mathrm{l}} / \mathrm{r}^{2}$, fonction des conditions aux limites, doit être nulle à l'état initial et, éventuellement, à l'infini, si on considère $\rho \rightarrow+\infty$.

Le terme entre parenthèses ne pouvant s'annuler on doit donc avoir :

$$
\frac{\mathrm{d} \sigma}{\mathrm{dr}}=0 \quad \text { C.Q.F.D. }
$$

Plus généralement encore, on démontre de la même façon que cette propriété reste vraie même si on ne suppose plus le matériau incompressible. $E^{\prime}(\sigma)$ et $\nu^{\prime}(\sigma)$ étant les coefficients élastiques en déformation plane, fonctions quelconques de $\sigma$, on a à la place de (A.15):

$$
\begin{gathered}
0=\frac{\mathrm{d} \sigma}{\mathrm{d} r}\left\{2-\frac{\mathrm{u}}{\mathrm{r}}\right. \\
\frac{\mathrm{d} \mathrm{E}^{\prime}(\sigma)}{\mathrm{d} \sigma}+\frac{\mathrm{d} \nu^{\prime}(\sigma)}{\mathrm{d} \sigma}\left(\sigma_{\mathrm{r}}-\sigma_{\mathrm{e}}\right) \\
\left.-\sigma_{e} \Phi(\sigma)\right\}
\end{gathered}
$$

où $\Phi(\sigma)$ est une fonction donnée, caractéristique du matériau :

$$
\Phi(\sigma)=\frac{1-2 \nu\left(\sigma_{e}\right)}{E\left(\sigma_{e}\right)} \frac{d}{d \sigma}\left(\frac{E(\sigma)}{1-\nu(\sigma)}\right)+\frac{d \nu^{\prime}(\sigma)}{d \sigma}
$$

A l'état initial $u / r=0$ et $\sigma_{\mathrm{r}}=\sigma=\sigma_{e}$; l'expression entre crochets de (A.16) ne peut s'annuler, sauf pour une valeur très particulière de $\sigma_{e}=\sigma_{e}^{*}$ :

$$
0=2-\sigma_{e}^{*} \Phi\left(\sigma_{e}^{*}\right)
$$

on doit donc avoir $\mathrm{d} \sigma / \mathrm{dr}=0$, pour tout chargement $\sigma_{e} \neq \sigma_{e}^{\circ}$.

Cette propriété reste vraie pour $\sigma_{e}=\sigma_{e}^{*}$ si on admet que $\sigma$ est une fonction continue du paramètre de chargement $\sigma_{e}$. 